\title{
COMPLEX POWERS OF THE LAPLACE OPERATOR ON THE CIRCLE
}

\author{
JACK MORAVA
}

\begin{abstract}
The classical zeta function of Lerch has an analytic continuation as a distribution on the circle which seems to be very different from its usual analytic continuation; for example, the Bernoulli polynomials come out upside down.
\end{abstract}

In this note functions on the circle $\mathbf{T}=\mathbf{R} / \mathbf{Z}$ are identified with periodic distributions on the line, and $\tilde{L}^{2}(\mathbf{T}, \mathbf{C})=L^{2}(\mathbf{T}, \mathbf{C}) / \mathbf{C}$ is the reduced Lebesgue space of nonconstant square-integrable periodic functions. This Hilbert space has the wellknown basis

$$
\mathbf{e}(n x)=e^{2 \pi i n x}, \quad n \in Z-\{0\}, x \in[0,1] .
$$

The complex powers of the Laplace operator [10] define an analytic group $(-\Delta)^{-s}$ of operators on $\tilde{L}^{2}(\mathbf{T}, \mathbf{C})$, and if $\sigma=$ re $s$ is sufficiently large, the prescription $(-\Delta)^{-s / 2} \mathbf{e}(n x)=n^{-s} \mathbf{e}(n x)$ leads to the integral representation

$$
(-\Delta)^{-s / 2} \phi(x)=\int_{0}^{1} K_{s}(x, y) \phi(y) d y
$$

for these complex powers by the kernel

$$
K_{s}(x, y)=\sum_{n \neq 0} n^{-s} \mathbf{e}(n x) \mathbf{e}(-n y) .
$$

Note that

$$
K_{s}(x, y)=1(s, x-y)+1(s, y-x),
$$

where

$$
\mathfrak{l}(s, x)=\sum_{n \geqslant 1} \mathbf{e}(n x) n^{-s}
$$

is a quite classical function. (See [14, §9.7, Example 2]; in particular, if $x \notin \mathbf{Z}$, then it has an analytic continuation, and-since for $x \neq 0,(d / d x)(s, x)=2 \pi i l(s-1, x)$-it is smooth away from the origin.) Now by Schwartz's kernel theorem [9] there is an analytic function of $s$, taking values in the distribution on $T \times T$ which are smooth away from the diagonal, representing $(-\Delta)^{-s / 2}$ for all $s$ in $C$. In particular, we can think of $K_{s}$ as taking values in the distributions on the line smooth away from the origin. The result in this paper is a formula for this kernel modulo functions smooth at the origin.

Received by the editors March 19, 1984 and, in revised form, June $11,1984$.

1980 Mathematics Subject Classification. Primary 10H10, 58G15, 81A19. 
Proposition. In some neighborhood of 0 in $\mathbf{T}$,

$$
\mathfrak{l}(s, x) \equiv \Gamma(1-s)(-2 \pi i x+0)^{s-1}
$$

in the group of distribution-valued meromorphic functions of $s$, modulo smooth-valued meromorphic functions of $s$.

This congruence requires some explanation, for the left side of the equation (defined on $\mathbf{T}$ ) is holomorphic, while the right side (defined on $\mathbf{R}$ ) has simple poles at the positive integers; but the residues at the poles turn out to be smooth. Otherwise, the Proposition is a straightforward consequence of a functional equation for a closely related function derived in 1887 by M. Lerch [5] which identifies 1 in terms of the Hurwitz zeta function

$$
\zeta(s, a)=\sum_{n \geqslant 0}(n+a)^{-s} .
$$

Note that

$$
-\Gamma(1-s) \theta^{s-1}=(s-1)^{-1}+(\gamma+\log \theta)+\text { positive powers of }(s-1)
$$

(cf. [2, I, §4.5]) where $\gamma$ is Euler's constant. Note also that, as $x \rightarrow 0,1(s, x) \rightarrow \zeta(s)$, for $\sigma>1$, but $\mathfrak{x}(s, x) \rightarrow+\infty$ when $\sigma \leqslant 1$; thus, to get the 'genuine' zeta function for re $s \leqslant 1$, it is necessary to add infinite counterterms, as in the Proposition. This is by now a hallowed procedure in physics (cf. [8, III, \$5 and IV, §5.1]). Hence,

$$
\mathfrak{l}(s, x)-\Gamma(1-s)(-2 \pi i x+0)^{s-1} \rightarrow \zeta(s) \text { as } x \rightarrow 0 \text { for all } s .
$$

COROLlary. $\mathfrak{Y}(1, x) \equiv-\log (-2 \pi i x)$ modulo smooth functions vanishing at 0 .

Similarly, $Y(s)$ represents a Hilbert-Schmidt operator on $\tilde{L}^{2}(\mathbf{T}, \mathbf{C})$ precisely when $Y(s)$ is in $L^{2}(\mathbf{T}, \mathbf{C})$-in particular, if $\sigma>1 / 2$.

Besides Lerch's equation we need to recall a few facts about the Feynman power $(x-i 0)^{s}$ from [2, I, §3.6]: this is an entire distribution-valued function of $s$, equal to the distribution limit as $\varepsilon \rightarrow+0$ of the functions $(x-i \varepsilon)^{s}$ or to the sum $x_{+}^{s}+$ e $\left(-\frac{1}{2} s\right) x_{-}^{s}$, where the (meromorphic, distribution-valued) functions $x_{ \pm}^{s}$ are as in Gel'fand-Šilov, or to the operation defined on test functions $f \in C_{c}^{\infty}$ by

$$
\int_{\mathbf{R}}(x-i 0)^{s} f(x) d x=\int_{L} z^{s} F(z) d z
$$

where $L$ is a contour running along the real axis from left to right, avoiding the origin by a small bump into the lower half-plane, and $F$ is an analytic extension of $f$ to some small neighborhood of $L$; Cauchy's theorem guarantees this operation to be well defined.

Now Lerch writes

$$
\Re(a, x, s)=\sum_{n \geqslant 0} \mathbf{e}(n x)(n+a)^{-s},
$$


with $a \in(0,1]$ and $\sigma>1$, and shows that an analytic continuation exists if $x \neq 0$ (or $1)$, verifying the functional equation

$$
\begin{aligned}
\Re(a, x, 1-s)=(2 \pi)^{-s} \Gamma(s)[ & \Re(x,-a, s) \mathbf{e}\left(\frac{1}{4} s-a x\right) \\
& \left.+\Re(1-x, a, s) \mathbf{e}\left(-\frac{1}{4} s+a(1-x)\right)\right] .
\end{aligned}
$$

See [1] for two proofs, or [11, I, §5] for another, or [15, VII, §8] for a fourth.

Now $\mathfrak{l}(s, x)=\mathbf{e}(x) \Re(1, x, s)$, while

$$
\Re(x,-1, s)=\zeta(s, x) \text { and } \Re(1-x, 1, s)=\sum_{n \geqslant 1}(n-x)^{-s},
$$

so for $\sigma$ sufficiently large we have

$$
\mathfrak{1}(1-s, x)=(2 \pi)^{-s} \Gamma(s) \mathbf{e}\left(\frac{1}{4} s\right)\left[x^{-s}+\mathbf{e}\left(-\frac{1}{2} s\right)(1-x)^{-s}\right]+\text { terms smooth at } 0 .
$$

If $f \in C^{\infty}$ is supported in a small neighborhood of 0 , then for sufficiently negative $\sigma$,

$$
\int_{0}^{1}\left[x^{-s}+\mathbf{e}\left(-\frac{1}{2} s\right)(1-x)^{-s}\right] f(x) d x=\int_{-1}^{+1}\left(x_{+}^{-s}+\mathbf{e}\left(-\frac{1}{2} s\right) x_{-}^{s}\right) f(x) d x,
$$

since $f$ is periodic; but then the equality must be true for all $s$, and

$$
1(1-s, x)-\mathbf{e}\left(\frac{1}{4} s\right)(2 \pi)^{-s} \Gamma(s)(x-i 0)^{-s}
$$

is smooth for large $\sigma$, hence for all $s$. This kind of functional equation is explained nicely by the Kubert-Lang theory of distributions (cf. $[3,4,7])$, these being functions on (for example) $\mathbf{R} / \mathbf{Z}$ with values in an abelian group, satisfying (for any positive integer $N$ ) the relation

$$
N^{k} \sum_{n-1 \geqslant j \geqslant 0} \phi\left(x+\bar{N}^{-1} j\right)=\phi(N x)
$$

here $k$ is the degree of the distribution $\phi$. Thus $(x-i 0)^{s-1}$ satisfies the distribution identity of degree $1-s$, in the group of germs of generalized functions at 0 on the real line, modulo germs of smooth functions; similarly $\log x$ is a distribution of degree 0 , taking values in such a group of asymptotic expansions near 0 (cf. [6, Lemma 6]). The author suggests the term 'retribution' for distribution-valued distributions.

The formula above is a little more familiar when expressed in terms of the Fourier transform: it is easier to think of $\mathcal{Y}$ as taking its values in the algebra of functions on $T$ under pointwise multiplication than in the convolution algebra. In particular, the transform of $\Gamma(s)(x-i 0)^{-s}$ is the distribution-valued meromorphic function $2 \pi \mathbf{e}\left(\frac{1}{4} s\right) x_{+}^{s-1}$; in the operational calculus in which $f(x) g(-i \partial)$ is the operator $\phi, \psi \rightarrow \int f \phi(g \hat{\psi})^{\swarrow}$, the caret denoting Fourier transform, we have

$$
\mathfrak{l}(1-s)=\mathbf{e}\left(\frac{1}{2}(1-s)\right)(2 \pi)^{1-s}\left(-i \partial_{-}\right)^{s-1}
$$

which is to say that $1(s)$ is roughly the $-s$ th power of $((-1 / 2 \pi i) \partial)$. This suggests that the above Proposition could be reformulated as another

COROllaRY. $(-\Delta)_{T}^{-s / 2}-(-\Delta)_{\mathbf{R}}^{-s / 2} \equiv \zeta(s)$ modulo smooth functions vanishing at 0 .

Our cousins, the physicists, might paraphrase this formula by saying that the zeta function at zero, which is something like a zero-point energy, can be calculated by 
analytical renormalization (i.e., by detaching the polar terms from its Laurent expansion; cf. [13, I, §3E]) to be equal to Euler's constant; but perhaps the question of whether or not Euler's constant is physical can be left to Euler.

This paper began in conversation with Jim McClure about the holomorphic divided power operations $s \mapsto \gamma_{s} x_{+}=\Gamma(1+s)^{-1} x_{+}^{s}$; in these terms a third reformulation of the Proposition is the

Corollary. $1(s, x) \equiv \pi \csc \pi s \gamma_{s-1}(-2 \pi i x+0)$.

I would like to thank him, as well as Dinakar Ramakrishnan, and especially Takashi Ono, for help with these questions and many others along the way. The assertions about physics above may not be very satisfying to physicists, but I would like to thank David Brydges and I. M. Singer for their time. Barry Simon, in helpful correspondence, points out that if $s=\sigma+i t$, with $\sigma$ strictly between zero and one, then $1(s)$ lies in the weak trace ideal $\mathscr{F}_{\sigma^{-1}, w}$ (cf. [12, I, viii]). Its Calderón norm is easy to calculate; we state it as a final

Corollary. $\|(s)\|_{\sigma^{-1}, w}=(1-\sigma)^{-1}$ if $s=\sigma+i t, \sigma \in(0,1)$.

\section{REFERENCES}

1. B. Berndt, Two new proofs of Lerch's functional equation, Proc. Amer. Math. Soc. 32 (1972), 403-408.

2. I. M. Gel'fand and G. E. Šilov, Generalised functions, Vol. I, Academic Press, New York, 1964.

3. D. Kubert and S. Lang, Modular units, Grundlehren Math. Wiss., Band 244, Springer, Berlin, 1981.

4. S. Lang, Units and class groups in number theory and algebraic geometry, Bull. Amer. Math. Soc. (N.S.) 6 (1982), 253-316 (appendix).

5. M. Lerch, Zakladove theorie Malmstenouskych rad, Rozpravy Československé Akad. Cišure Frantiska Josefa 27 (1892), 525-592.

6. J. Milnor, Polylogarithms, the Hurwitz zeta-function and the Kubert identities, Enseign. Math. 29 (1983), 281-322.

7. D. Ramakrishnan, On the monodromy of higher logarithms, Proc. Amer. Math. Soc. 85 (1982), 596-599.

8. P. Ramond, Field theory: a modern primer, Princeton Frontiers in Physics 57 (1981).

9. L. Schwartz, Théorie des distributions, Vol. II, Hermann, Paris, 1966.

10. R. T. Seeley, Complex powers of elliptic operators, Proc. Sympos. Pure Math., Vol. 10, Amer. Math. Soc., Providence, R.I., 1967, pp. 288-308.

11. C. L. Siegel, Lectures on advanced analytic number theory, Tata Institute, Bombay, 1961.

12. B. Simon, Trace ideals and applications, London Math. Soc. Lecture Notes 35, Cambridge Univ. Press, 1979.

13. E. R. Speer, Generalized Feynman amplitudes, Ann. of Math. Stud., Vol. 62, Princeton Univ. Press, Princeton, N. J., 1969.

14. E. C. Titchmarsh, Theory of functions, Oxford Univ. Press, New York and London, 1932.

15. A. Weil, Elliptic functions according to Kronecker and Eisenstein, Springer-Verlag, Berlin and New York, 1976.

Department of Mathematics, Johns Hopkins University, Baltimore, Maryland 21218 